\title{
Bariatrische Chirurgie bei Jugendlichen: Kapitulation vor dem Lebensstil
}

\begin{abstract}
Massiv übergewichtige Jugendliche erreichen mit einem maximalen diätetischen Aufwand nur in etwa $10 \%$ eine Reduktion der überschüssigen Pfunde um die Hälfte. Nach Anlage eines Magenbandes erreichen $\mathbf{8 4 \%}$ dieses Ziel.
\end{abstract}

— Im Jahr 2004 waren 17,4\% der amerikanischen Jugendlichen adipös, vier Jahre zuvor erst $14,8 \%$. Der Trend scheint ungebrochen, und plötzlich

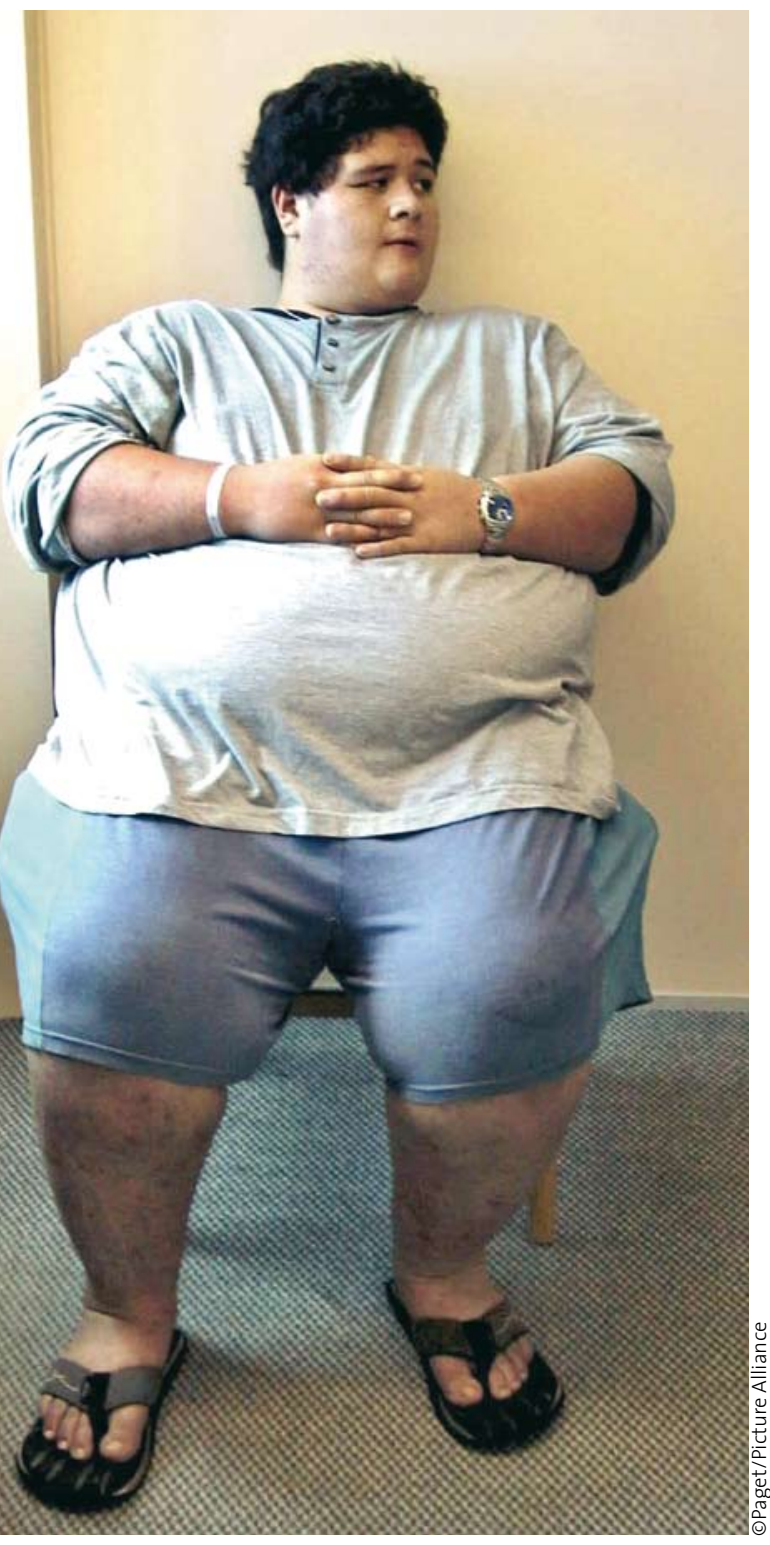

haben es Kinderärzte mit Erkrankungen zu tun, die bisher in dieser Altersgruppe völlig unbekannt waren: metabolisches Syndrom, Typ-2-Diabetes, Hypertonie, Dyslipidämie, polyzystisches Ovarsyndrom und nicht alkoholische Steatohepatitis. Nachdem sich konservative diätetische Programme als weitgehend unwirksam erwiesen haben, ruhen nun die Hoffnungen auf der bariatrischen Chirurgie.

Zwischen 2005 und 2008 wurden in Melbourne/Australien 14-18-jährige Jugendliche mit einem Body-MassIndex über $35 \mathrm{~kg} / \mathrm{m}^{2}$ zur Teilnahme an einer randomisierten Studie eingeladen, die den Effekt eines assistierten Programms zur Veränderung des Lebensstils mit den Auswirkungen der Anlage eines Magenbandes vergleichen sollte. In der auf zwei Jahre angelegten Studie wurden das Körpergewicht, die Parameter des metabolischen Syndroms, eine Insulinresistenz, die Lebensqualität und unerwünschte Begleitwirkungen erfasst.

24 von 25 Jugendlichen in der Magenbandgruppe und 18 von 25 in der Lebensstilgruppe beendeten die Studie wie vorgesehen. Nach der Anlage des Magenbandes verloren 21 von 25 übergewichtigen Jugendlichen mehr als 50\% des überschüssigen Gewichts, in der ebenso großen Gruppe mit der Veränderung des Lebensstils waren es dagegen ganze drei. Der durchschnitt-

Bei derart massivem Übergewicht muss schon bei Jugendlichen zu extremen Maßnahmen gegriffen werden. liche Gewichtsverlust nach Anlage des Magenbandes belief sich auf Werte zwischen 30,2 und 39,0 kg. In der Lebensstilgruppe lagen diese Werte zwischen 2,1 und $8,1 \mathrm{~kg}$.

Neun Studienteilnehmer in der bariatrisch behandelten Gruppe und zehn in der Lebensstilgruppe erfüllten die Kriterien des metabolischen Syndroms. Nach zwei Jahren war bei keinem der bariatrisch behandelten Probanden ein metabolisches Syndrom nachweisbar, in der konservativ behandelten Kontrollgruppe jedoch noch bei vier von 18 Probanden. Perioperative Komplikationen oder eine Einschränkung der Lebensqualität traten bei keinem Probanden aus der Magenbandgruppe auf. Allerdings waren bei sieben Probanden im Lauf der Nachbeobachtung insgesamt acht Operationen wegen lokaler Komplikationen erforderlich.

\section{Kommentar}

Das Übergewicht scheint für viele Menschen der Preis für den modernen Lebensstil zu sein. Es mag auf den ersten Blick befremdlich erscheinen, wenn wir dieses Problem nun mit einer schnellen technologischen Lösung beseitigen wollen. Wahrscheinlich ist es aber kurzfristig der einzig gangbare Weg und für Jugendliche deshalb besonders wichtig, weil massives Übergewicht in dieser prägenden Lebensphase das gesamte zukünftige Schicksal erheblich beeinflusst. Ob wir als Gesellschaft in der Lage sind, auf freiwilliger $B a-$ sis wieder zu einem gesünderen Lebensstil zurückzukehren, ist fraglich. In jedem Fall dauert es aber für die heute betroffenen Jugendlichen zu lange.

H.-S. FÜEßL =

\footnotetext{
- P. E. O'Brien et al.

(Korr. : Paul E. O'Brien, MD, Centre for Obesity Research and Education, Monash University Medical School, Alfred Hospital, Melbourne, 3004, Australia, e-mail:paul.obrien@med. monash.edu.au): Laparoscopic adjustable gastric banding in severely obese adolescents. JAMA 303 (2010) 6, 519-526
} 